\title{
Bone Marrow Suppression Secondary to the COVID-19 Booster Vaccine: A Case Report
}

\author{
Toral Shastri', Navkiran Randhawa ${ }^{2}$, Ragia $\mathrm{Aly}^{3}$, Masood Ghouse ${ }^{3}$ \\ 'Chicago College of Osteopathic Medicine, Midwestern University, Downers Grove, IL, USA; ${ }^{2}$ Franciscan Health, Olympia Fields, IL, USA; ${ }^{3}$ The \\ Department of Hematology and Oncology, Franciscan Health, Olympia Fields, IL, USA \\ Correspondence: Toral Shastri, Tel +630 440-0655, Email tshastri96@midwestern.edu
}

\begin{abstract}
As of September 2021, SARS-CoV-2 booster shots became widely available in the US to ensure continued protection against the virus. A temporal relationship has been previously reported between the first or second dose of the COVID-19 vaccine and the development of thrombocytopenia. However, adverse events related to the third COVID-19 vaccine are still being reported and studied. We report a 74-year-old male who developed bone marrow suppression and pancytopenia recorded seven days after receiving the Pfizer SARS-CoV-2 vaccine. During his hospital stay, the patient's hemoglobin, white blood cell, and platelet levels continued to trend downwards. However, all three levels showed improvement one week after discharge without robust intervention. Global vaccination is of utmost importance, as is understanding and documenting post-vaccination reactions including bone marrow suppression. Prompt evaluation and patient education are imperative to improve patient outcomes and combat hesitancy against vaccine administration.
\end{abstract}

Keywords: COVID, pancytopenia, vaccination, Pfizer, bone marrow suppression

\section{Introduction}

Since its emergence in December of 2019, the rapid spread of severe acute respiratory syndrome coronavirus (SARSCoV-2) has quickly affected millions of lives across every continent. ${ }^{1}$ This highly transmittable and pathogenic viral infection has led to massive mitigation efforts and allocation of resources to prevent the spread of transmission and high mortality related to complications. ${ }^{2}$ The establishment of higher levels of community (herd) immunity and protection against SARS-CoV-2 via the widespread deployment of effective vaccines has become a global effort. ${ }^{3}$ In December of 2020, the FDA issued an Emergency use Authorization for the Pfizer-BioNTech and Moderna COVID-19 Vaccine as a two-dose series. ${ }^{4}$ In September 2021, booster vaccines became widely administered in the US due to waning immunity of the COVID-19 vaccines against variants of SARS-CoV-2 along with ensuring continued protection against the virus. ${ }^{5}$

Serious adverse events such as anaphylaxis, Guillain-Barre Syndrome, myocarditis, pericarditis, thrombocytopenia, and death have been previously reported following the first and/or second dose of vaccine. ${ }^{6}$ To our knowledge, no cases have been reported regarding bone marrow suppression related to the third COVID-19 vaccine. Adverse events reported between August 12-September 19, 2021 from the COVID-19 booster vaccine supported similar reactions to those after dose two. ${ }^{7}$ According to the Centers for Disease Control and Prevention (CDC), these initial findings indicate no unexpected patterns of adverse reactions after an additional dose of COVID-19 vaccination. ${ }^{7}$ However, adverse events related to the COVID-19 booster are still being reported and studied. ${ }^{6}$ This report presents a case of bone marrow suppression occurring after the third COVID-19 vaccine without a similar reaction after the first or second dose.

\section{Case Report}

A 74-year-old male with a history of polychondritis and hypothyroidism presented to the hospital after falling out of his chair and inability to ambulate. The patient was found to be mildly confused upon arrival to the emergency room, limiting our ability to obtain a full verbal history. Chart review revealed the patient had received his third Pfizer Covid 
vaccine shot seven days before admission followed by fatigue, decreased appetite, fever, and chills. The patient had received the second Pfizer Covid-19 shot nine months before the booster. No reactions to the previous two shots were noted.

Upon initial evaluation, vital signs were within normal limits and a physical exam revealed significant tenderness in the upper arm and no gross bleeding (Figure 1). Computed tomography (CT) imaging (Figure 2) was significant for enhancement of the left axillary lymph node. The patient's initial complete blood count (CBC) was remarkable for a hemoglobin count of $9.9 \mathrm{~g} / \mathrm{dl}$ and platelet count of $84 \times 10^{9} / \mathrm{L}$; both values lower than his prior hemoglobin count of $13.7 \mathrm{~g} / \mathrm{dl}$ and platelet count of $180 \times 10^{9} / \mathrm{L}$ from December of 2020 . His mean corpuscular volume (MCV) was elevated at 101.3 femtolitres from his prior MCV value of 95.8 femtolitres in December of 2020. His white blood cell (WBC) count was recorded at $7.6 \times 10^{9} / \mathrm{L}$.

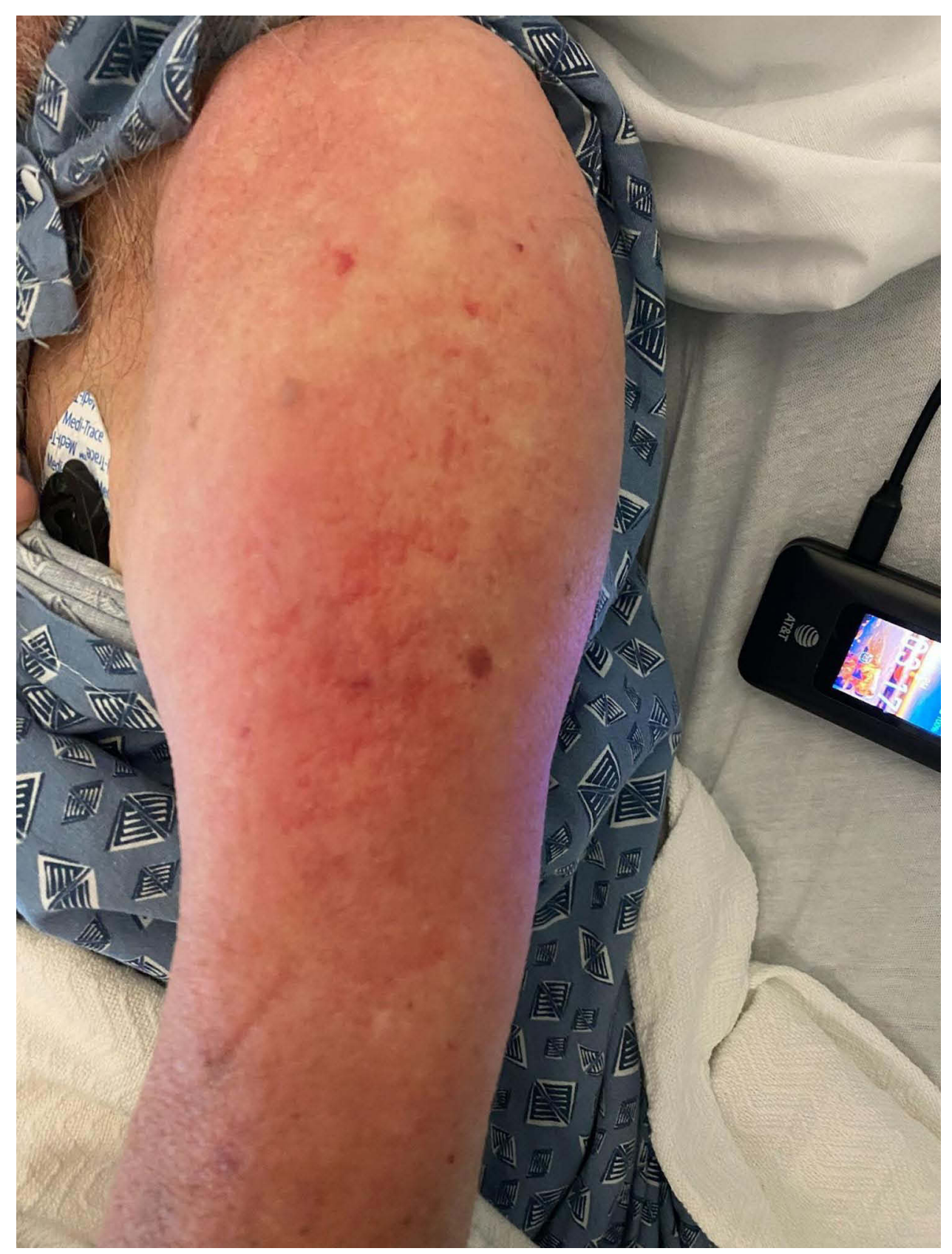

Figure I The patient's upper arm showed erythema with no gross bleeding near the injection site 


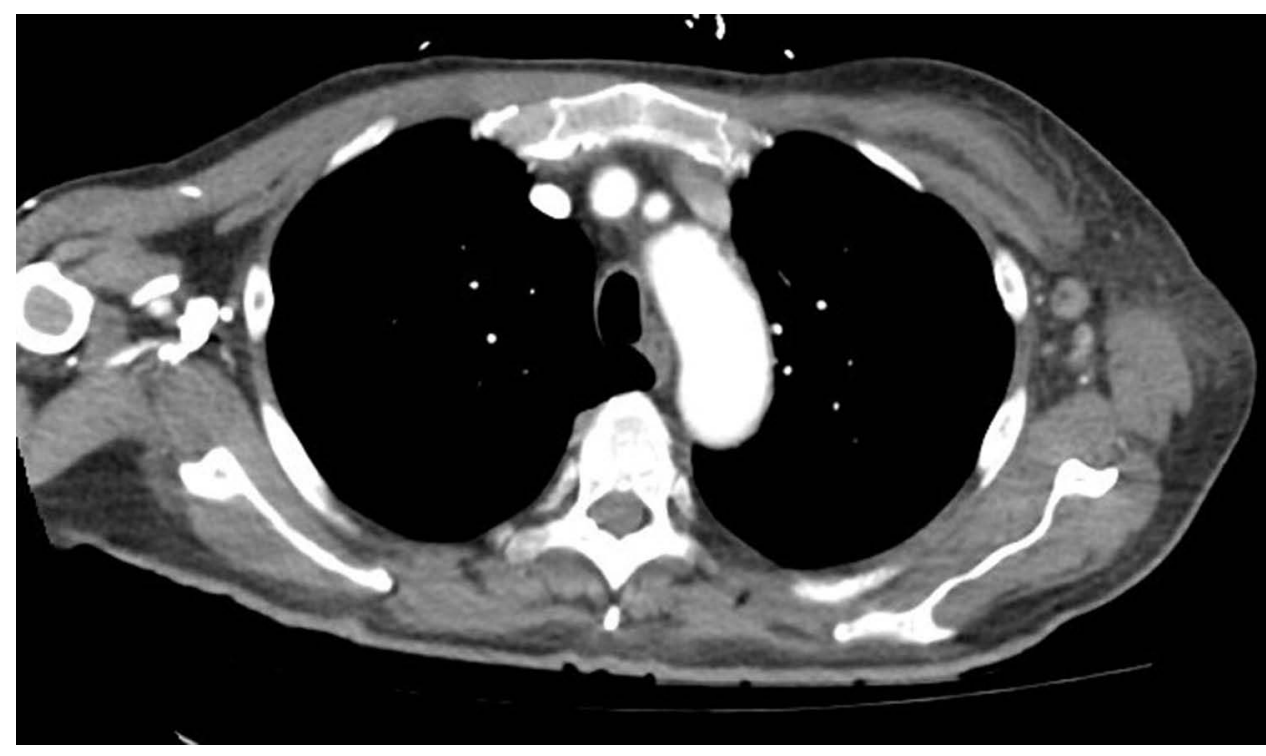

Figure 2 The patient's CT imaging of the thoracic region showed enhancement of the left axillary lymph node.

The hemoglobin, WBC, and platelet count further down trended from his baseline (Figures 3-5). Anemia labs including ferritin levels $(554 \mathrm{ng} / \mathrm{mL})$, vitamin B12 $(253 \mathrm{pg} / \mathrm{mL})$, total bilirubin $(0.5 \mathrm{mg} / \mathrm{dL})$, and reticulocyte count $(0.8 \%)$ were nonsignificant during the patient's hospital stay. The patient's left shoulder presented with extensive bruising, erythema, papular rash, warmth, and tenderness on palpation during the hospitalization. An improvement in WBC and platelet levels was noted on day 4 of hospitalization.

Before discharge, the patient was fully alert and oriented and reported improvement in his symptoms. Examination of his lateral left arm showed decreased erythema and bruising with slight petechiae. The patient was discharged due to stabilization of labs and encouraged to take oral vitamin B12 supplements. During his outpatient follow-up six days after hospitalization, his hemoglobin increased to $10.5 \mathrm{~g} / \mathrm{dl}$, WBC count increased to $4.9 \times 10^{9} / \mathrm{L}$, and platelets increased to $101 \times 10^{9} / \mathrm{L}$.

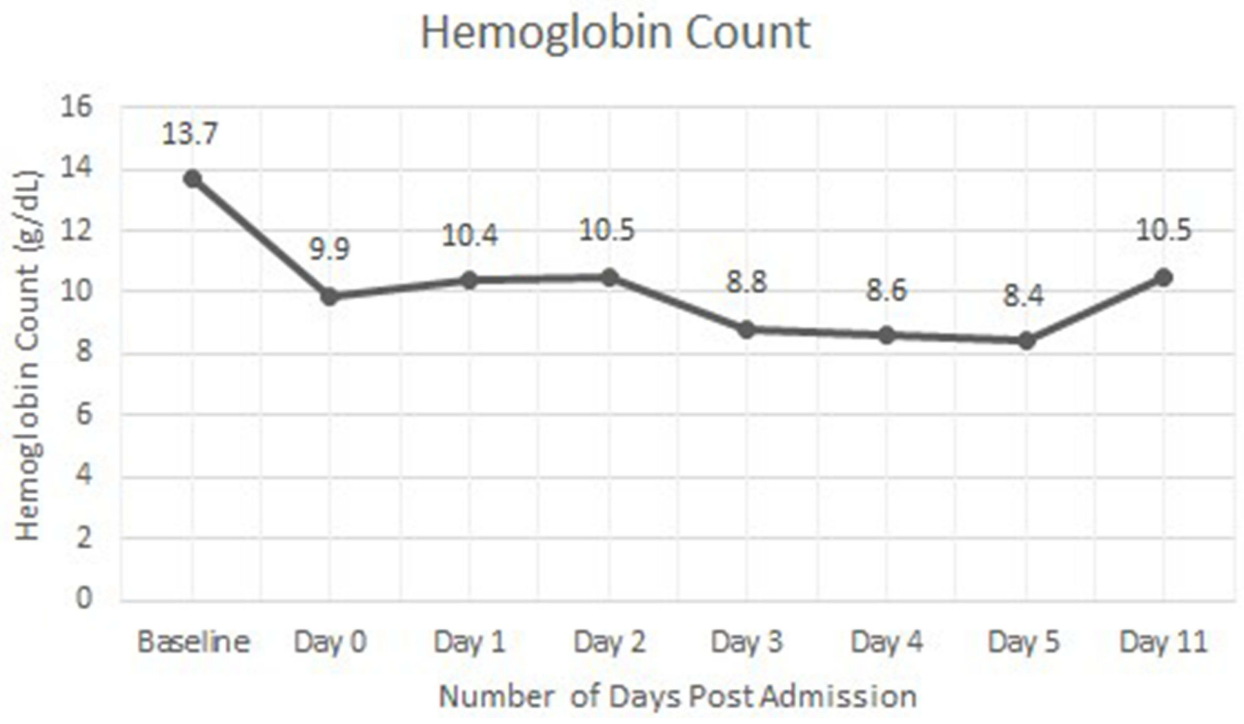

Figure 3 The patient's hemoglobin count throughout his hospital course and 6 days after discharge. 


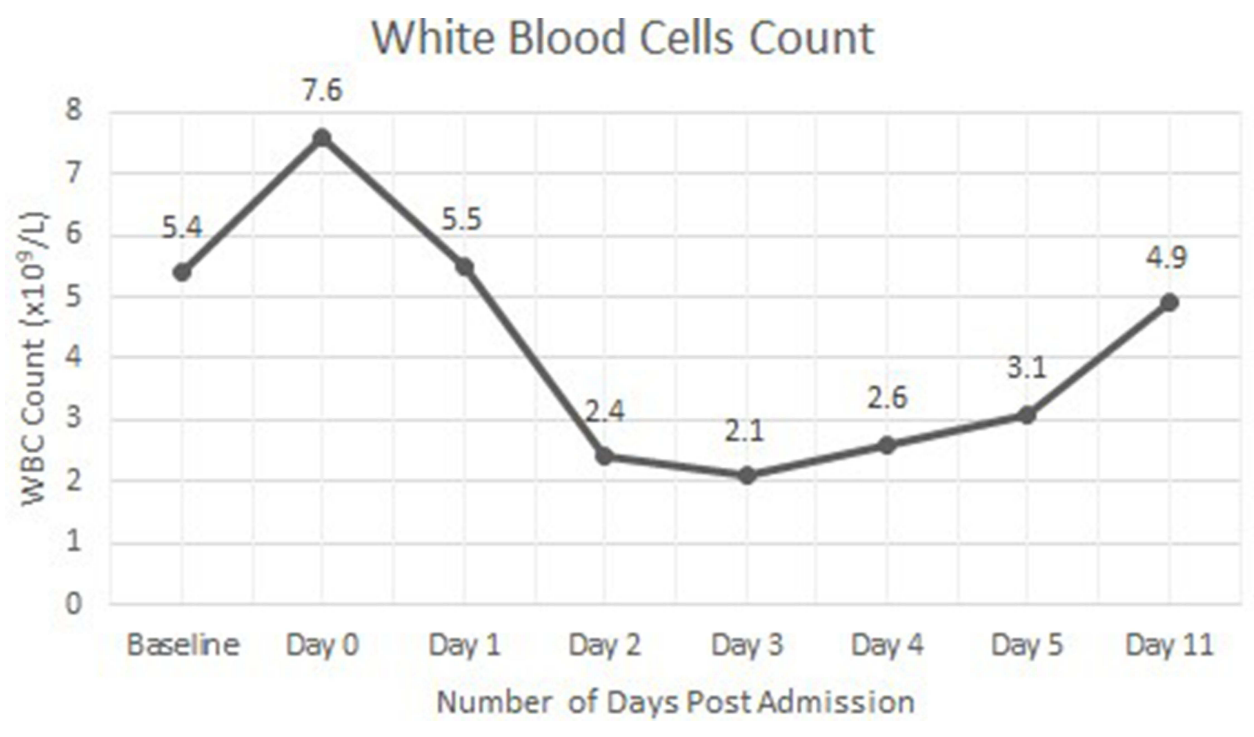

Figure 4 The patient's WBC count throughout his hospital course and 6 days after discharge.

Platelet Count

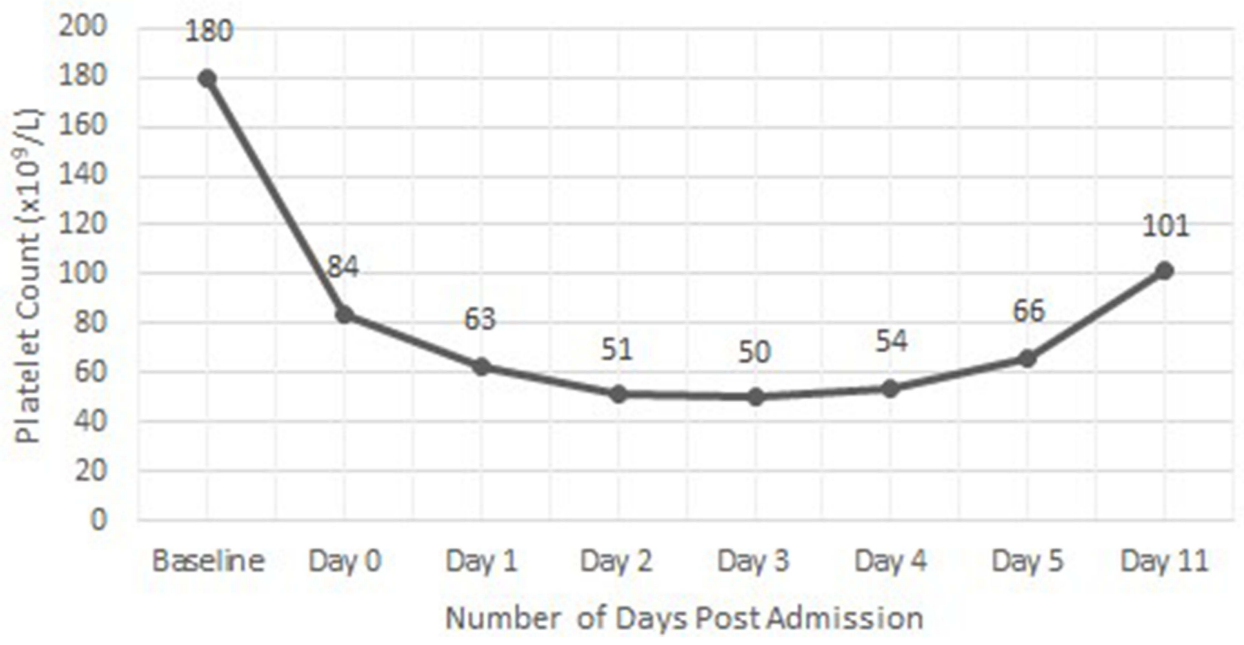

Figure 5 The patient's platelet count throughout his hospital course and 6 days after discharge.

\section{Discussion}

This paper presents a patient with pancytopenia recorded seven days after receiving the Pfizer booster vaccine. Interestingly, this patient did not report any reactions after the first or second dose of the Pfizer vaccine against SARSCoV-2. Pancytopenia refers to a decrease in all peripheral bloodlines and is present when all three cell lines are below the normal reference range. ${ }^{8}$ The patient's physical exam showed no signs of active bleeding along with his labs indicating no evidence of hemolysis. The patient's hemoglobin, platelet, and white blood cell count presented below baseline followed by a decrease and slight improvement during his hospital stay. Six days after hospitalization, all three cell lines showed improvement. The temporal association with the booster vaccine and negative infectious disease workup raised suspicion for vaccine-induced bone marrow suppression. In addition, the patient's reticulocyte count and lactate dehydrogenase value were consistent with hypoproliferation within the bone marrow.

Currently, there is a gap in knowledge of adverse events specific to the third vaccine against SARS-CoV-2 due to the recent initiation of administration and ongoing reporting of events. ${ }^{6}$ To our knowledge, bone marrow suppression after 
any dose of vaccine against SARS-CoV-2 has not been previously reported. However, a prior case of pancytopenia after the third vaccination with a recombinant hepatitis B vaccine has previously been reported. ${ }^{9}$ The patient's bone marrow biopsy within this case displayed a paucity of late myeloid elements and CD8+ T cells. ${ }^{9}$ It was believed the patient's CD8 $+\mathrm{T}$ cells were causing excessive production of IFN- $\gamma$; a stimulant of negative regulators of hematopoiesis such as tumor necrosis factor and lymphotoxin. ${ }^{10}$ IFN- $\gamma$ has also previously been reported to create immunological effects comprising an upregulation of histocompatibility gene transcription and alteration in class I and II antigen expression at the cell surface. ${ }^{11}$ It was predicted these changes resulted in an autoimmune reaction causing suppression of maturation of hematopoietic progenitor cells and pancytopenia. ${ }^{9}$ Via a similar mechanism, we believe that our patient's pancytopenia was immune-mediated, potentially triggered by the vaccination.

Vaccines against SARS-CoV-2 (first or second dose) and the induction of Idiopathic Thrombocytopenic Purpura (ITP) have also been recently acknowledged in multiple cases. ${ }^{12}$ Our patient presented with low platelet levels and associated petechiae and purpura at the site of the vaccination. However, the patient's presentation of low hemoglobin and white blood cells along with normal reticulocyte levels was more indicative of pancytopenia secondary to bone marrow suppression. In patients presenting with pancytopenia, the history and the physical exam should help assess the severity of the pancytopenia and comorbid illnesses that may complicate the disorder. ${ }^{13}$ In addition, suspicious medications and exposure to toxic agents should be ruled out. ${ }^{13}$ Initial screening laboratory evaluation should include the patient's complete blood count, peripheral blood smear examination, reticulocyte count, complete metabolic panel, prothrombin time/partial thromboplastin time, and blood type and screen. Common interventions to alleviate bone marrow suppression and pancytopenia include treating the underlying cause and utilizing supplements to boost red blood cell production if indicated.

Vaccines against SARS-CoV-2 undergo continuous safety monitoring; adverse events are very rare. ${ }^{14}$ However, vaccine hesitancy remains a barrier towards full population inoculation against SARS-CoV-2 and is influenced by misinformation regarding vaccine safety. ${ }^{15}$ One study using an anonymous online questionnaire found a person's trust in the effectiveness of the vaccine was a major facilitative factor affecting willingness to vaccinate. ${ }^{16}$ The same study also found that $66.7 \%$ of unvaccinated participants thought the vaccine's safety was not enough, making it the main reason for reluctance or hesitance to be vaccinated. ${ }^{16}$ Therefore, education of adverse events and available interventions post-vaccination is imperative to prevent the spread of misinformation and combat hesitancy towards vaccination. ${ }^{15}$

As of September 19, 2021, about 2.2 million people in the United States received a third vaccine against SARS-CoV $-2 .{ }^{17}$ Among those who received the vaccine, 22,000 people reported the effects of the vaccine with no unexpected patterns of adverse reactions. ${ }^{17}$ Our patient demonstrates abnormal pancytopenia first recorded seven days after receiving the booster vaccine, possibly indicating a rare adverse event from the vaccination given the temporal relationship. While additional studies and observations are indicated to verify bone marrow suppression as an adverse reaction, this case report provides an opportunity for patient education and treatment planning before symptoms arise.

\section{Conclusion}

Our case report highlights pancytopenia secondary to bone marrow suppression following Pfizer vaccination against SARS-CoV-2. It is important to consider the possibility of bone marrow suppression following the third vaccine against SARS-CoV-2. Although additional studies are indicated to determine the risk factors and pathogenesis of vaccine-induced bone marrow suppression, prompt evaluation and initiation of interventions can improve patient outcomes.

\section{Consent for Publication}

Institutional approval was not required to publish the case details. The publication of this study has been consented to by the patient.

\section{Disclosure}

The authors report no conflicts of interest in this work. 


\section{References}

1. Fernandes A, Chaudhari S, Jamil N, Gopalakrishnan G. COVID-19 vaccine. Endocr Pract. 2021;27(2):170-172. doi:10.1016/j.eprac.2021.01.013

2. Johansson MA, Quandelacy TM, Kada S, et al. SARS-CoV-2 transmission from people without COVID-19 symptoms. JAMA Network Open. 2021;4(1):e2035057-e2035057. doi:10.1001/jamanetworkopen.2020.35057

3. Graham BS. Rapid COVID-19 vaccine development. Science. 2020;368(6494):945-946. doi:10.1126/science.abb8923

4. Gee J, Marquez P, Su J, et al. First month of COVID-19 vaccine safety monitoring-United States, December 14, 2020-January 13. Morb Mortal Wkly Rep. 2021;70(8):283. doi:10.15585/mmwr.mm7008e3

5. Mahase E. Covid-19 booster vaccines: what we know and who's doing what. BMJ. 2021. doi: 10.1136/bmj.n2082

6. Centers for Disease Control and Prevention. Selected adverse events reported after COVID-19 vaccination. Centers for Disease Control and Prevention. Available from: https://www.cdc.gov/coronavirus/2019-ncov/vaccines/safety/adverse-events.html. Accessed November 8, 2021.

7. Hause AM. Safety monitoring of an additional dose. Centers for Disease Control and Prevention; 2021. Available from: https://www.cdc.gov/ mmwr/volumes/70/wr/mm7039e4.htm. Accessed February 11, 2022.

8. Valent P. Low blood counts: immune mediated, idiopathic, or myelodysplasia. Hematology. 2012;2012(1):485-491. doi:10.1182/asheducation. V2012.1.485.3798522

9. Viallard JF, Boiron JM, Parrens M, et al. Severe pancytopenia triggered by recombinant hepatitis B vaccine. Br J Haematol. 2000;110(1):230-233. doi:10.1046/j.1365-2141.2000.02171.x

10. Collart MA, Belin D, Vassalli JD, De Kossodo S, Vassalli P. Gamma interferon enhances macrophage transcription of the tumor necrosis factor/ cachectin, interleukin 1, and urokinase genes, which are controlled by short-lived repressors. J Exp Med. 1986;164(6):2113-2118. doi:10.1084/ jem.164.6.2113

11. Wallach D, Fellous M, Revel M. Preferential effect of gamma interferon on the synthesis of HLA antigens and their mRNAs in human cells. Nature. 1982;299(5886):833-836. doi:10.1038/299833a0

12. Shah SRA, Dolkar S, Mathew J, et al. COVID-19 vaccination associated severe immune thrombocytopenia. Exp Hematol Oncol. $2021 ; 10: 42$. doi:10.1186/s40164-021-00235-0

13. Elizabeth P, Weinzierl MD, Daniel A, Arber MD. The differential diagnosis and bone marrow evaluation of new-onset pancytopenia. Am J Clin Pathol. 2013;139(1):9-29. doi:10.1309/AJCP50AEEYGREWUZ

14. Centers for Disease Control and Prevention. COVID-19 vaccination; 2020. Available from: https://www.cdc.gov/coronavirus/2019-ncov/vaccines/ safety/safety-of-vaccines.html. Accessed February 11, 2022.

15. Dror AA, Eisenbach N, Taiber S, et al. Vaccine hesitancy: the next challenge in the fight against COVID-19. Eur J Epidemiol. 2020;35:775-779. doi:10.1007/s10654-020-00671-y

16. Gan L, Chen Y, Hu P, et al. Willingness to receive SARS-CoV-2 vaccination and associated factors among Chinese adults: a cross sectional survey. Int J Environ Res Public Health. 2021;18(4):1993. doi:10.3390/ijerph18041993

17. STAT. Early data suggest side effects after Covid booster similar to second dose; 2021. Available from: https://www.statnews.com/2021/09/28/sideeffect-rates-from-a-third-covid-19-vaccine-dose-similar-to-those-after-second-shot-early-data-indicate/. Accessed February 11, 2022.

Journal of Blood Medicine

\section{Publish your work in this journal}

The Journal of Blood Medicine is an international, peer-reviewed, open access, online journal publishing laboratory, experimental and clinical aspects of all aspect pertaining to blood based medicine including but not limited to: Transfusion Medicine; Blood collection, Donor issues, Transmittable diseases, and Blood banking logistics; Immunohematology; Artificial and alternative blood based therapeutics; Hematology; Biotechnology/nanotechnology of blood related medicine; Legal aspects of blood medicine; Historical perspectives. The manuscript management system is completely online and includes a very quick and fair peer-review system. Visit http://www.dovepress.com/testimonials.php to read real quotes from published authors.

Submit your manuscript here: http://www.dovepress.com/journal-of-blood-medicine-journal 